\title{
New therapeutic protocol for improvement of endometrial receptivity (PRIMER) for patients with recurrent implantation failure (RIF) - A pilot study
}

Felipe Dieamant ${ }^{1,2}$, Laura D. Vagnini ${ }^{2}$, Claudia G. Petersen ${ }^{1,2}$, Ana L. Mauri ${ }^{1,2}$, Adriana Renzi ${ }^{2}$, Bruna Petersen ${ }^{1,2}$, Mariana C. Mattila ${ }^{1}$, Andreia Nicoletti ${ }^{1,2}$, João Batista A. Oliveira ${ }^{1,2}$, Ricardo Baruffi ${ }^{1,2}$, Jose G. Franco $\mathrm{Jr}^{1,2}$

${ }^{1}$ Center for Human Reproduction Prof Franco Jr. Ribeirão Preto, Brazil

${ }^{2}$ Paulista Center for Diagnosis Research and Training. Ribeirão Preto, Brazil

Presented at the $74^{\text {th }}$ Scientific Congress of the American Society for Reproductive Medicine, 2018, Denver, Colorado.

\begin{abstract}
Objective: To evaluate whether or not one should use a new Protocol for Endometrial Receptivity Improvement (PRIMER) based on platelet-rich plasma (PRP) and granulocyte colony-stimulation factor (G-CSF) to enhance ongoing pregnancy rates in patients with recurrent implantation failure (RIF).

Methods: Women undergoing IVF/ICSI were prospectively divided into two groups: - PRIMER/RIF group $(n: 33)$ : patients with RIF (defined as $\geq 2$ embryo transfers (ETs) and at least 5 morphologically good embryos transferred) in which intrauterine PRP injection and subcutaneous G-CSF-injection were performed. - Control group ( $\mathrm{n}: 33$ ): patients in their first IVF/ICSI attempt/cycle (without PRP or G-CSF injection). The PRP was prepared using autologous fresh-whole blood processed to increase platelet-concentration in 2 to 4 fold. All patients undergoing the PRP-treatment received $0.7 \mathrm{ml}$ of it through intrauterine-injection 48 hours before the ET. G-CSF $(300 \mathrm{mg} / 0.5 \mathrm{ml})$ started simultaneously to PRP and was administered subcutaneously every week.

Results: Regarding implantation, clinical pregnancy and miscarriage rates, we found no statistically significant difference $(18.2 \%$ versus $17.6 \%, p=0.90 ; 36.4 \%$ versus $30.3 \%, p=0.61$ and $25.0 \%$ versus $9.0 \%, p=0.43$, respectively). The use of PRIMER enabled RIF patients (previous ET $\mu: 4.0 \pm 1.5$ ) to reach similar ongoing pregnancy and live birth rates like those patients who had their first IVF/ICSI cycle attempt $(27.3 \%$ versus $27.3 \%, p=0.99)$.

Conclusions: Our results showed, for the first time, evidence that this therapeutic protocol (PRIMER) could be used as a feasible treatment based on biological rationale for patients with RIF, considering its promising outcomes, it is a simple procedure and not associated with patient complications.
\end{abstract}

Keywords: recurrent implantation failure, endometrial receptivity, therapeutic protocol, granulocyte colonystimulation factor, platelet-rich plasma, PRIMER

\section{INTRODUCTION}

The success of a treatment in assisted reproductive technique (ART) cycles depends on the perfect synchrony between embryonic development and endometrial receptivity. The implantation process requires endometrial growth and differentiation of endometrial stromal cells. Human endometrium contains growth factors, receptors for growth factors, cytokines, and other key factors for correct embryonic and endometrial development. Multiple embryos fail to implant, and a relevant percentage of IVF/ ICSI treatment failures are due to endometrial receptivity disorders (Blois et al., 2011; Farimani et al., 2017).

Recurrent implantation failure (RIF) is a common concern among researchers, physicians and patients. Couples desperately require further diagnostic investigations and/or the use of adjunctive/alternative treatments in order to improve their pregnancy likelihoods. In fact, RIF has been the target of a wide scientific debate and several approaches have been developed in order to solve this reproduction issue (Bos-Mikich et al., 2019). Endometrial injury, intrauterine human chorionic gonadotrophin (hCG), endometrial receptivity array (ERA), Preimplantation genetic testing for aneuploidy (PGT-A), "Omics" tools (Genomics, Transcriptomics, Proteomics, Metabolomics), and other techniques have been used in patients with RIF, but the results found still require further analyses for routine use in IVF/ICSI cycles (Somigliana et al., 2018; Nardo et al., 2015; Spencer et al., 2016; Macklon, 2017; Hviid \& Macklon, 2017. The granulocyte colony-stimulating factor (G-CSF) and Platelet-rich Plasma (PRP) are among these new therapeutic approaches for RIF.

PRP is prepared from fresh whole blood and contains several growth factors and cytokines, including vascular endothelial growth factor (VEGF), transforming growth factor (TGF), platelet-derived growth factor (PDGF) and epidermal growth factor (EGF). Therefore, it may help regulate endometrial cell migration, attachment, proliferation, differentiation, and neoangiogenesis, resulting in beneficial effects on endometrial receptivity. The use of Platelet-rich Plasma (PRP) in human reproduction is growing, and it could be a new tool to improve clinical outcomes in patients undergoing ART procedures (Zeyneloglu \& Onalan, 2014; Chang et al., 2015; Magdi et al., 2017). PRP has been used in assisted reproduction, especially in patients with thin endometria (Dhillon et al., 2012; Lee et al., 2013).

G-CSF is a hematopoietic lineage-specific cytokine, associated with cell proliferation and differentiation, produced by reproductive tissue cells. This cytokine promotes endometrial immunomodulation and optimizes the interaction between the embryo and the endometrium. Studies have shown that its use is associated with higher pregnancy rates and lower miscarriage rates (Lédée et al., 2013; Rahmati et al., 2015; Arefi et al., 2018).

Considering the beneficial effects of PRP and G-CSF on endometrial receptivity, in addition to the fact that failures in IVF/ICSI cycles are related to endometrial disorders, we conducted a pilot study to evaluated if a new therapeutic Protocol for Improvement of Endometrial Receptivity 
(PRIMER) based on the use of PRP and G-CSF together can improve ART outcomes in patients with RIF.

\section{MATERIAL AND METHODS}

\section{Population}

A total of 66 patients enrolled in the IVF/ICSI program at the Prof Franco Jr. Center for Human Reproduction, from February 2017 to October 2017, and they were prospectively included in this study. We obtained a complete medical/surgical history from all patients, and total screening was performed to exclude RIF causes (a normal karyotype for her and her partner, and no evidence of uterine defects, ultrasonographic evidence of hydrosalpinx, infections, endocrine problems, coagulation defects, thrombophilia and autoimmune defects). We considered only one fresh embryo transfer cycle. We excluded transfer cycles of frozen embryos.

The women were divided into two groups:

-PRIMER/RIF group: patients with RIF in which intrauterine PRP injection and subcutaneous G-CSF injection were performed. RIF was defined as $\geq 2$ embryo transfers (ET), and at least 5 good-morphological embryos were transferred.

-Control group: patients in their first IVF/ICSI cycle attempt (without PRP or G-CSF).

The two groups were matched using the Key Performance Indicators Score (Franco Jr et al., 2017) based on female-age, anti-Müllerian hormone (AMH) levels, number of metaphase-II oocytes, fertilization rates, and morphological quality of embryos-transferred.

\section{Ovarian stimulation protocol}

All patients included in the study were submitted to the same ovarian stimulation protocol: the long gonadotropin releasing hormone ( $\mathrm{GnRH}$ ) agonist protocol (GnRH-a) as previously described (Oliveira et al., 2012). The starting FSH dose was based on the patient's age, anti-Müllerian hormone level and antral follicle count (Ovarian Response Prediction Index calculation) (Oliveira et al., 2012).

To induce final oocyte maturation we administered $250 \mu \mathrm{g}$ of recombinant human chorionic gonadotropin (r-hCG; Ovidrel; Serono, Brazil) subcutaneously when at least two follicles reached a mean diameter of $\geq 17 \mathrm{~mm}$. GnRH-a was administered until the day of the r-hCG injection. Oocytes were retrieved by a transvaginal aspiration under ultrasound guidance, 34-36 hours following the r-hCG injection.

\section{ART procedures}

All metaphase II oocytes received ICSI, which was carried out as previously described (Mauri et al., 2010; Oliveira et al., 2011). The oocytes were examined after 17$20 \mathrm{~h}$ to assess fertilization; zygotes with two distinct equalsized pronuclei were considered normal.

The embryos were routinely transferred after $96 \mathrm{~h}$ in culture, and supernumerary embryos were cryopreserved. The embryos were then transferred with a Frydman catheter (Frydman ${ }^{\circledR}$ Classic Catheter 4.5 CCD Laboratoire C.C.D; Paris, France) guided by abdominal ultrasound, using a 3.5-MHz convex transducer (Aloka SSD-1100; Aloka Co. Ltd, Tokyo, Japan). A single physician performed all embryo transfers, and only easy transfers (i.e. the catheter passed smoothly through the cervix without the need for uterine fixation clamps) with clear visualization of the catheter tip upon ultrasound were considered. All the patients received luteal phase supplementation with vaginal natural progesterone (Utrogestan ${ }^{\circledR}$; Besins Healthcare, São Paulo, Brazil).

\section{Platelet-rich Plasma}

PRP was prepared using autologous fresh whole blood and a double-spin method. In brief, blood was obtained by venipuncture in syringes containing acid citrate dextrose $(A C D-A)$ solution $(1: 4 \mathrm{vol} / \mathrm{vol})$. The citrated blood was centrifuged for $12 \mathrm{~min}$ at $1400 \mathrm{rpm}$. Subsequently, the supernatant plasma was taken up using a micropipette and transferred into another sterile tube for centrifugation. A second round of centrifugation was performed for $7 \mathrm{~min}$ at $3200 \mathrm{rpm}$. Platelets pellet formed at the bottom of the tube. The supernatant was removed in another sterile tube and the platelets were suspended in a minimum quantity of plasma by gently shaking the tube. The process increases platelet-concentration in 2 to 4 fold.

All patients undergoing the PRP-treatment (study group) received $0.7 \mathrm{ml}$ of it through intrauterine injection, using a soft catheter, 48 hours before the ET.

\section{Granulocyte colony-stimulating factor}

On the same day of the PRP injection, we injected G-CSF (Filgrastim ${ }^{\circledR}$, Biosintetica/Achê, $300 \mu \mathrm{g} / 0.5 \mathrm{ml}$ ) subcutaneously, and repeated it weekly. If pregnancy occurs, $\mathrm{G}-\mathrm{CSF}$ is maintained until the $12^{\text {th }}$ gestation week.

\section{Endpoints}

The primary endpoints were ongoing pregnancy and live birth rates. The secondary endpoints included implantation, clinical pregnancy and miscarriage rates.

\section{Statistical analysis}

Data management and univariate analysis were carried out using the StatsDirect statistical software version 2.7.9 software (Cheshire, UK). The following parameters were evaluated: the woman's age, infertility etiology, number of oocytes retrieved, number of oocytes in metaphase II retrieved fertilization rate, the number of embryos transferred, embryo implantation rates, miscarriage rates, ongoing pregnancy rates and live birth rates. We used the nonparametric Mann-Whitney test to compare the means of continuous variables, when the continuous variables were not normally distributed, and the Student's t-test was used if the continuous variables were normally distributed. The results are expressed as the arithmetic means \pm standard deviation (SD). For categorical variables, we used the Fisher's exact test to check between group associations, and the results were expressed as percentages. A $p$ value $<0.05$ was considered statistically significant.

\section{RESULTS}

We found an equal distribution $(p>0.05)$ of the general and cycle's characteristics for PRIMER/RIF (PRP injection and subcutaneous G-CSF injection) and Control (without PRP or G-CSF) groups. Table 1 summarizes the data.

There were no significant differences between the PRIMER and Control groups regarding implantation, pregnancy, spontaneous miscarriage, ongoing pregnancy or live birth rates $p>0.05$ ). Table 2 shows the main results.

\section{DISCUSSION}

Our results indicated that the PRIMER enabled patients with RIF (mean number of previous ET of $4.0 \pm 1.5$ ) to reach similar ongoing pregnancy and live birth rates to those patients who had their first IVF/ICSI cycle attempt, demonstrating possible beneficial effects on endometrial receptivity. Unfortunately, to the best of our knowledge, the present study is the first to analyze the efficacy of intrauterine PRP associated with subcutaneous G-CSF for RIF patients, and thus cannot be compared with other results. 
Table 1. General and cycles' characteristics

\begin{tabular}{|c|c|c|c|}
\hline & PRIMER & Control & $\boldsymbol{p}$ \\
\hline $\mathrm{n}$ & 33 & 33 & \\
\hline Previous ET (n) & $4.0 \pm 1.5$ & ---- & \\
\hline Previous embryos transferred (n) & $9.1 \pm 2.1$ & ---- & \\
\hline Female age $(y)$ & $37.8 \pm 3.8$ & $37.8 \pm 3.9$ & 0.94 \\
\hline Male age $(y)$ & $41.8 \pm 4.8$ & $39.1 \pm 6.2$ & 0.10 \\
\hline $\mathrm{AMH}(\mathrm{ng} / \mathrm{dl})$ & $2.4 \pm 2.7$ & $2.7+4.7$ & 0.43 \\
\hline BMI $\left(\mathrm{Kg} / \mathrm{m}^{2}\right)$ & $24.4 \pm 3.3$ & $24.8 \pm 3.9$ & 0.23 \\
\hline $\begin{array}{l}\text { Etiology of infertility } \\
\text {-Male factor (\%) } \\
\text {-Tubal peritoneal (\%) } \\
\text {-Idiopathic (\%) } \\
\text {-Endometriosis (\%) }\end{array}$ & $\begin{array}{c}42.4 \%(14 / 33) \\
12.2 \%(4 / 33) \\
24.2 \%(8 / 33) \\
21.2 \%(7 / 33) \\
\end{array}$ & $\begin{array}{c}36.4 \%(12 / 33) \\
9.1 \%(3 / 33) \\
33.3 \%(11 / 33) \\
21.2 \%(7 / 33) \\
\end{array}$ & 0.62 \\
\hline FSH total dose (IU) & $3245.9 \pm 1357.7$ & $2866.4 \pm 1125.8$ & 0.23 \\
\hline LH total dose (IU) & $1398.3 \pm 151.2$ & $1084.8 \pm 160.4$ & 0.30 \\
\hline Oocytes retrieved $(n)$ & $7.8 \pm 4.4$ & $6.4 \pm 3.6$ & 0.14 \\
\hline MII oocytes (n) & $6.1 \pm 3.5$ & $5.3 \pm 3.1$ & 0.35 \\
\hline Fertilization rate \% & $60.6 \pm 23.2$ & $68.2 \pm 25.5$ & 0.14 \\
\hline embryos transferred $(n)$ & $2.3 \pm 0.9$ & $2.2 \pm 0.9$ & 0.53 \\
\hline Good quality embryo transferred (\%) & $54.5 \%(18 / 33)$ & $60.6 \%(20 / 33)$ & 0.62 \\
\hline
\end{tabular}

Table 2. Main results

\begin{tabular}{|l|c|c|c|}
\hline & PRIMER & Control & P \\
\hline Implantation rate (\%) & $18.2 \%(14 / 77)$ & $17.6 \%(12 / 68)$ & 0.90 \\
\hline Clinical pregnancy rate (\%) & $36.4 \%(12 / 33)$ & $30.3 \%(10 / 33)$ & 0.61 \\
\hline Miscarriage rate (\%) & $25.0 \%(3 / 12)$ & $9.0 \%(1 / 10)$ & 0.43 \\
\hline Ongoing pregnancy rate (\%) & $27.3 \%(9 / 33)$ & $27.3 \%(9 / 33)$ & 0.99 \\
\hline Live birth rate (\%) & $27.3 \%(9 / 33)$ & $27.3 \%(9 / 33)$ & 0.99 \\
\hline
\end{tabular}

However, PRP and G-CSF have been employed singly in several medical fields, including human reproduction, with promising outcomes, corroborating our findings.

Previous trials have shown the beneficial effects of PRP use on tissues, promoting cell-differentiation, proliferation, growth, and neoangiogenesis (Aghajanova et al., 2016). These effects are probably because it contains and/ or stimulates several growth factors such as TGF- $\beta$, PDGF, IGF, VEGF, EGF and FGF-2 and bioactive-cytokines, which stimulate the inflammatory cascade and the healing process (Le et al., 2019). These PRP positive effects could improve reproductive outcomes in patients with impaired endometrial receptivity. However, few trials have evaluated the effectiveness of autologous PRP in patients with endometrium disorders (Chang et al., 2015; Tandulwadkar et al., 2017; Zadehmodarres et al., 2017; Eftekhar et al., 2018; Molina et al., 2018; Mehrafza et al., 2019).

Regarding the use of G-CSF, studies have shown that its use could improve endometrial thickness and pregnancy rates in patients presenting suboptimal endometria (Jain et al., 2018). G-CSF is a glycoprotein, secreted from endothelial cells, macrophages and some other immune system cells. Recently, it has been used in assisted reproduction for patients presenting thin endometrium and/or recurrent pregnancy loss. Some studies have demonstrated that the G-CSF use by transvaginal endometrial perfusion or subcutaneous way could improve ART outcomes, such as implantation and miscarriage rates (Würfel et al., 2010; Zafardoust et al., 2017; Zhang et al., 2018). However, the results in the general population undergoing ART are questionable (Barad et al., 2014; Jain et al., 2018).

Considering PRP is obtained through fresh-whole blood peripheral vein, its intrauterine-injection is relatively safe, with low-risk of disease-transmission, immune reactions, and deleterious effects to the patient (Welte, 2014). On the other hand, besides G-CSF beneficial effects on reproductive tissues, it is considered safe within the dose and route of administration usually employed (Jang et al., 2017). Therefore, the combined use of subcutaneous G-CSF and intrauterine PRP - PRIMER - should be considered as low risk for IVF/ICSI patients. In addition, because of the action of these growth factors and bioactive-cytokines, PRIMER could have beneficial effects on endometrial receptivity.

A limitation of this study is that it is not randomized. However, the KPI-score (female-age, anti-Müllerian hormone $(A M H)$ levels, number of metaphase-II oocytes, fertilization rate, and morphological quality of embryos-transferred) and cycles characteristics including male-age, FSH total dose, mean number of oocytes retrieved, mean number of embryos-transferred, and etiology of infertility found in the study population were similar between the Study and Control groups. These findings confirm that the groups were adequately matched, minimizing bias in the study outcomes. 
In conclusion, our results showed, for the first time, evidence that this therapeutic protocol (PRIMER) could be used as a feasible treatment based on biological rationale for patients with RIF, since these patients with about four previous ETs achieved ongoing pregnancy rates similar to patients who performed their first IVF/ICSI attempt cycle. The use of a rigorous match based on the KPI-score provides relevance to the outcomes found. In order to confirm the results found in this study, randomized controlled trials in a large population should be carried out.

\section{CONFLICT OF INTEREST}

The authors declare no conflict of interest.

\section{Corresponding author:}

Jose G. Franco Jr.

Centre for Human Reproduction Prof Franco Jr.

Ribeirão Preto, SP, Brazil.

E-mail: crh@crh.com.br

\section{REFERENCES}

Aghajanova L, Houshdaran S, Balayan S, Irwin J, Huddleston $H$, Giudice $L$. Platelets for endometrial regeneration: a novel approach. Fertil Steril. 2016;106:e82. DOI: 10.1016/j.fertnstert.2016.07.241

Arefi S, Fazeli E, Esfahani M, Borhani N, Yamini N, Hosseini A, Farifteh F. Granulocyte-colony stimulating factor may improve pregnancy outcome in patients with history of unexplained recurrent implantation failure: An RCT. Int J Reprod Biomed (Yazd). 2018;16:299-304.PMID: 30027145 DOI: $10.29252 /$ ijrm.16.5.299

Barad DH, Yu Y, Kushnir VA, Shohat-Tal A, Lazzaroni E, Lee $\mathrm{HJ}$, Gleicher N. A randomized clinical trial of endometrial perfusion with granulocyte colony-stimulating factor in in vitro fertilization cycles: impact on endometrial thickness and clinical pregnancy rates. Fertil Steril. 2014;101:710-5. PMID: 24424357 DOI: 10.1016/j.fertnstert.2013.12.016

Blois SM, Klapp BF, Barrientos G. Decidualization and angiogenesis in early pregnancy: unravelling the functions of DC and NK cells. J Reprod Immunol. 2011;88:86-92. PMID: 21227511 DOI: $10.1016 /$ j.jri.2010.11.002

Bos-Mikich A, Ferreira MO, de Oliveira R, Frantz N. Platelet-rich plasma or blood-derived products to improve endometrial receptivity? J Assist Reprod Genet. 2019 [Epub ahead of print] PMID: 30610660 DOI: $10.1007 / \mathrm{s} 10815-018-1386-\mathrm{z}$

Chang Y, Li J, Chen Y, Wei L, Yang X, Shi Y, Liang X. Autologous platelet-rich plasma promotes endometrial growth and improves pregnancy outcome during in vitro fertilization. Int J Clin Exp Med. 2015;8:1286-90. PMID: 2578512

Dhillon RS, Schwarz EM, Maloney MD. Platelet-rich plasma therapy - future or trend? Arthritis Res Ther. 2012;14:219. PMID: 22894643 DOI: 10.1186/ar3914

Eftekhar M, Neghab N, Naghshineh E, Khani P. Can autologous platelet rich plasma expand endometrial thickness and improve pregnancy rate during frozen-thawed embryo transfer cycle? A randomized clinical trial. Taiwan J Obstet Gynecol. 2018;57:810-3. PMID: 30545532 DOI: 10.1016/j.tjog.2018.10.007
Farimani M, Poorolajal J, Rabiee S, Bahmanzadeh M. Successful pregnancy and live birth after intrauterine administration of autologous platelet-rich plasma in a woman with recurrent implantation failure: A case report. Int J Reprod Biomed (Yazd). 2017;15:803-6.PMID: 29492478 DOI: 10.29252/ijrm.15.12.803

Franco JG Jr, Petersen CG, Mauri AL, Vagnini LD, Renzi A, Petersen B, Mattila MC, Comar VA, Ricci J, Dieamant F, OIiveira JBA, Baruffi RLR. Key performance Indicators score (KPIs-score) based on clinical and laboratorial parameters can stablish benchmarks for internal quality control in an ART program. JBRA Assist Reprod. 2017;21:61-6. PMID: 28609268 DOI: 10.5935/1518-0557.20170016

Hviid MM, Macklon N. Immune modulation treatments-where is the evidence? Fertil Steril 2017;107:1284-93. PMID: 28501364 DOI: 10.1016/j.fertnstert.2017.04.009

Jain S, Mahey R, Malhotra N, Kalaivani M, Sangeeta P, Bhatt A, Singh N, Kriplani A. Effect of Intrauterine Perfusion of Granulocyte Colony-stimulating Factor on Endometrial Parameters and In Vitro Fertilization Outcome in Women Undergoing In Vitro Fertilization/Intracytoplasmic Sperm Injection Cycles: A Randomized Controlled Trial. J Hum Reprod Sci. 2018;11:25460. PMID: 30568355 DOI: 10.4103/jhrs.JHRS_20_18

Jang HY, Myoung SM, Choe JM, Kim T, Cheon YP, Kim YM, Park H. Effects of Autologous Platelet-Rich Plasma on Regeneration of Damaged Endometrium in Female Rats. Yonsei Med J. 2017;58:1195-203. PMID: 29047244 DOI: 10.3349/ymj.2017.58.6.1195

Le ADK, Enweze L, DeBaun MR, Dragoo JL. Platelet-Rich Plasma. Clin Sports Med. 2019;38:17-44. PMID: 30466721 DOI: $10.1016 /$ j.csm.2018.08.001

Lédée N, Gridelet V, Ravet S, Jouan C, Gaspard O, Wenders F, Thonon F, Hincourt N, Dubois M, Foidart JM, Munaut C, Perrier d'Hauterive S. Impact of follicular G-CSF quantification on subsequent embryo transfer decisions: a proof of concept study. Hum Reprod. 2013;28:406-13.PMID: 23223438 DOI: $10.1093 /$ humrep/des354

Lee JW, Kwon OH, Kim TK, Cho YK, Choi KY, Chung HY, Cho BC, Yang JD, Shin JH. Platelet-rich plasma: quantitative assessment of growth factor levels and comparative analysis of activated and inactivated groups. Arch Plast Surg. 2013;40:5305. PMID: 24086805 DOI: 10.5999/aps.2013.40.5.530

Macklon N. Recurrent implantation failure is a pathology with a specific transcriptomic signature. Fertil Steril. 2017;108:914.PMID: 28602479 DOI: 10.1016/j.fertnstert.2017.05.028

Magdi Y, El-Damen A, Fathi AM, Abdelaziz AM, Abd-Elfatah Youssef M, Abd-Allah AA, Ahmed Elawady M, Ahmed Ibrahim $M$, Edris $Y$. Revisiting the management of recurrent implantation failure through freeze-all policy. Fertil Steril. 2017; 108:727. PMID: 28579406 DOI: 10.1016/j.fertnstert.2017.04.020

Mauri AL, Petersen CG, Oliveira JB, Massaro FC, Baruffi RL, Franco JG Jr. Comparison of day 2 embryo quality after conventional ICSI versus intracytoplasmic morphologically selected sperm injection (IMSI) using sibling oocytes. Eur J Obstet Gynecol Reprod Biol. 2010;150:42-6. PMID: 20171776 DOI: $10.1016 /$ j.ejogrb.2010.01.004 
Mehrafza M, Kabodmehri R, Nikpouri Z, Pourseify G, Raoufi A, Eftekhari A, Samadnia S, Hosseini A. Comparing the impact of autologous platelet-rich plasma and granulocyte colony stimulating factor on pregnancy outcome in patients with repeated implantation failure. J Reprod Infertil. 2019;20:35-41. PMID: 30859080

Molina A, Sánchez J, Sánchez W, Vielma V. Platelet-rich plasma as an adjuvant in the endometrial preparation of patients with refractory endometrium. JBRA Assist Reprod. 2018;22:42-8. PMID: 29303234 DOI: 10.5935/1518-0557.20180009

Nardo LG, El-Toukhy T, Stewart J, Balen AH, Potdar N. British Fertility Society Policy and Practice Committee: adjuvants in IVF: evidence for good clinical practice. Hum Fertil (Camb). 2015;18:2-15. PMID: 25531921 DOI: 10.3109/14647273.2015.985454

Oliveira JB, Cavagna M, Petersen CG, Mauri AL, Massaro FC, Silva LF, Baruffi RL, Franco JG Jr. Pregnancy outcomes in women with repeated implantation failures after intracytoplasmic morphologically selected sperm injection (IMSI). Reprod Biol Endocrinol. 2011;9:99. PMID: 21781299 DOI: 10.1186/1477-7827-9-99

Oliveira JB, Baruffi RL, Petersen CG, Mauri AL, Nascimento AM, Vagnini L, Ricci J, Cavagna M, Franco JG Jr. A new ovarian response prediction index (ORPI): implications for individualized controlled ovarian stimulation. Reprod Biol Endocrinol. 2012;10:94. PMID: 23171004 DOI: 10.1186/1477-7827-10-94

Rahmati $M$, Petitbarat $M$, Dubanchet $S$, Bensussan $A$, Chaouat G, Ledee N. Colony Stimulating Factors 1, 2, 3 and early pregnancy steps: from bench to bedside. J Reprod Immunol. 2015;109:1-6. PMID: 25721620 DOI: 10.1016/j.jri.2015.01.005

Somigliana E, Vigano P, Busnelli A, Paffoni A, Vegetti W, Vercellini P. Repeated implantation failure at the crossroad between statistics, clinics and over-diagnosis. Reprod Biomed Online. 2018;36:32-8.PMID: 29102484 DOI: 10.1016/j.rbmo.2017.09.012

Spencer EA, Mahtani KR, Goldacre B, Heneghan C. Claims for fertility interventions: a systematic assessment of statements on UK fertility centre websites. BMJ Open. 2016;6:e013940. PMID: 27890866 DOI: 10.1136/bmjopen-2016-013940
Tandulwadkar SR, Naralkar MV, Surana AD, Selvakarthick $M$, Kharat AH. Autologous Intrauterine Platelet-Rich Plasma Instillation for Suboptimal Endometrium in Frozen Embryo Transfer Cycles: A Pilot Study. J Hum Reprod Sci. 2017;10:208-12. PMID: 29142450 DOI: 10.4103/jhrs. JHRS_28_17

Welte K. G-CSF: filgrastim, lenograstim and biosimilars. Expert Opin Biol Ther. 2014;14:983-93. PMID: 24707817 DOI: $10.1517 / 14712598.2014 .905537$

Würfel W, Santjohanser C, Hirv K, Bühl M, Meri O, Laubert I, von Hertwig I, Fiedler K, Krüsmann J, Krüsmann G. High pregnancy rates with administration of granulocyte colony-stimulating factor in ART-patients with repetitive implantation failure and lacking killer-cell immunglobulin-like receptors. Hum Reprod. 2010;25:2151-2. PMID: 20522442 DOI: 10.1093/humrep/deq106

Zadehmodarres S, Salehpour S, Saharkhiz N, Nazari L. Treatment of thin endometrium with autologous platelet-rich plasma: a pilot study. JBRA Assist Reprod. 2017;21:54-6. PMID: 28333034 DOI: 10.5935/15180557.20170013

Zafardoust S, Akhondi MM, Sadeghi MR, Mohammadzadeh A, Karimi A, Jouhari S, Ansaripour S. Efficacy of Intrauterine Injection of Granulocyte Colony Stimulating Factor (G-CSF) on Treatment of Unexplained Recurrent Miscarriage: A Pilot RCT Study. J Reprod Infertil. 2017;18:37985. PMID: 29201668

Zeyneloglu HB, Onalan G. Remedies for recurrent implantation failure. Semin Reprod Med. 2014;32:297-305. PMID: 24919029 DOI: $10.1055 / \mathrm{s}-0034-1375182$

Zhang L, Xu WH, Fu XH, Huang QX, Guo XY, Zhang L, Li SS, Zhu J, Shu J. Therapeutic role of granulocyte colony-stimulating factor (G-CSF) for infertile women under in vitro fertilization and embryo transfer (IVF-ET) treatment: a meta-analysis. Arch Gynecol Obstet. 2018;298:861-71. PMID: 30220024 DOI: 10.1007/s00404-018-4892-4 